\title{
LOWER BOUND OF THE HADWIGER NUMBER OF GRAPHS BY THEIR AVERAGE DEGREE
}

\author{
A. V. KOSTOCHKA
}

Received I February 1982

The aim of this paper is to show that the minimum Hadwiger number of graphs with average degree $k$ is $O(k / \sqrt{\log k})$. Specially, it follows that Hadwiger's conjecture is true for aimost all graphs with $n$ vertices, furthermore if $k$ is large enough then for almost all graphs with $n$ vertices and $n k$ edges.

\section{Introduction}

Let us recall the notion of the Hadwiger number. The following operations are called elementary contractions:

substitution of two adjacent points $v_{1}$ and $v_{2}$ for a new point $v_{3}$ connected to the points connected to $v_{1}$ or $v_{2}$;

removal of an edge;

removal of a point.

The graph $G$ is said to be contractable to the graph $H$ if $H$ can be obtained from $G$ by means of a sequence of elementary contractions. The Hadwiger number $\eta(G)$ of a graph $G$ is the maximum order of the complete graphs to which $G$ can be contracted.

A contraction of a connected graph $G=(V, E)$ to the complete graph of $r$ vertices also can be considered as an onto mapping $\psi: V \rightarrow\{1,2, \ldots, r\}$ with the following properties:

every subgraph $\psi^{-1}(i)$ of $G$ is connected $(1 \leqq i \leqq r)$

for any integers $1 \leqq i<j \leqq r$, there exist vertices $v \in \psi^{-1}(i)$ and $w \in \psi^{-1}(j)$ such that $(v, w) \in E$.

The contraction is one of the most natural operations on graphs. Thus, it is very important to study the Hadwiger number and its relation to other features of graphs. For example, let us mention Hadwiger's well-known conjecture ([2]) that the chromatic number $\chi(G)$ of a graph $G$ is not greater than $\eta(G)$. Let $w(k)=$ $=\min \{\eta(G): \chi(G) \geqq k\}$. Then Hadwiger's conjecture is equivalent to the following statement: $w(k)=k$ for any natural number $k$. Wagner [7] showed that $w(k)=$ $\geqq 4+\log _{2}(k-1 / 3)$. Mader's [5] result implies that $w(k)>(k / 16) \log _{2} k$.

AMS subject classification 1980): 05 C 10, 05 C 15, 60 C 05 . 
Many authors (see e.g. [5], [6], [8], [9]) are interested in how small Hadwiger number a graph $G=(V, E)$ can have if $|E| /|V| \geqq k$. Denote this minimum by $\eta(k)$. Considering contraction as mapping, we can easily prove that the Hadwiger number of almost all graphs of $n$ vertices is at most $n / \sqrt{\log n}$. It means that there exists a graph $G=(V, E)$ with $|V|=n,|E| \geqq n^{2} / 4$ and $\eta(G) \leqq n / \sqrt{\log n}$. Thus $\eta(k) \leqq 4 k / \sqrt{\log k}$ if $k$ is large enough. On the other hand, Mader [5] proved that $\eta(k)>k / 8 \log _{2} k$. That is, if $k$ is large then

$$
\frac{k}{8 \log _{2} k}<\eta(k) \leqq \frac{4 k}{\sqrt{\log k}} .
$$

The aim of this paper is to prove that the order of $\eta(k)$ is $O(k / \sqrt{\log k})$. The consequences of this statement improve the lower bound of $w(k)$ and prove Hadwiger's conjecture for for almost all graphs of $n$ vertices and $k n$ edges if $k$ is large enough.

\section{Notation}

The sign " $\doteq$ " must be read as "equal by definition".

Let $G=(V, E)$ be a graph and $V_{0} \subset V$. Then $G\left(V_{0}\right)$ and $G-V_{0}$ denote the subgraphs of $G$ induced by $V_{0}$ and $V-V_{0}$, resp. If $v \in V$ then $N_{G}(v) \doteq\{u \in V-\{v\}$ : $(u, v) \in E\}, \quad d_{G}(v) \doteq\left|N_{G}(v)\right|, \quad \sigma(G) \doteq \max _{v \in V} d_{G}(v), \quad \delta(G) \doteq \min _{v \in V} d_{G}(v)$. For a graph $G=$ $=(V, E), \bar{G}=(V, \bar{E})$ denotes the complement of $G$. Let $\mathscr{D}_{k} \doteq\{G=(V, E) ; \quad|E| /|V| \geqq$ $\geqq k\}$. Then $\eta(k)=\min _{G \in \mathscr{Q}_{k}} \eta(G)$.

\section{Results and comments}

Theorem 1. $\eta(k) \geqq k / 270 \sqrt{\log k}$ for $k \geqq 2$.

Corollary 2. $w(k) \geqq k / 540 \sqrt{\log k}$ for $k \geqq 2$.

Corollary 3. Hadwiger's conjecture is true for almost all graphs of $n$ vertices.

Note. Corollary 3 was proved by P. Erdős, B. Bollobás and P. A. Catlin independently from each other.

Corollary 4. If $k$ is large enough then Hadwiger's conjecture is true for almost all graphs of $n$ vertices and $k n$ edges.

Corollary 5. $\min _{G=(V, E),|V|=n}(\eta(G)+\eta(\bar{G}))=\Omega(n / \sqrt{\log n})$.

Let $v(k)$ be the smallest possible Hadwiger number of a $k$-connected graph. Mader [5] proved that $\eta(k) / 2 \leqq \nu(k) \leqq 2 \eta(k)$. From these inequalities and Theorem 1 , we obtain

Corollary 6. $v(k)=\Omega(k / \sqrt{\log k})$. 
Previously, the class $\varepsilon_{k}=\left\{G=(V, E):|V| \geqq k,|E|>k \cdot|V|-\left(\begin{array}{c}k+1 \\ 2\end{array}\right)\right\}$ and the function $\eta_{1}(k)=\min _{G \in \mathscr{\delta}_{k}} \eta(G)$ were studied instead of $\mathscr{D}_{k}$ and $\eta(k)$ for different reasons (see e.g. [5], [8], [9]). As it is a more natural class, we formulate our results for $\mathscr{D}_{k}$. Actually, we prove a bit stronger statement than Theorem 1:

Theorem 1'. $\eta_{1}(k) \geqq 1 / 270 \cdot(k / \sqrt{\log k})$ for $k \geqq 2$.

From the proof of Theorem $1^{\prime}$, it is easy to obtain a polynomial-time algorithm with the help of that any graph in $\varepsilon_{k}$ can be contracted to the complete graph of $k / 270 \sqrt{\log k}$ vertices.

We can see in the proof of Theorem $1^{\prime}$ that the constant $1 / 270$ can be improved at the expense of the length of the proof. It is proved in [4] that

$$
.064 \frac{k}{\sqrt{\log k}} \leqq \eta_{1}(k) \leqq 2.25 \frac{k}{\sqrt{\log k}} .
$$

The structure of the proof is as follows. In section 4, we prove that it is sufficient to study the contraction of graphs containing much more edges than its complement. Some features of contraction of such graphs are studied in section 5. Using these features, a lower bound of the Hadwiger number of such graphs is given in section 6 by an auxiliary function. Theorem $1^{\prime}$ is proved in section 7 on the basis of sections 4 and 6 . Corollaries $2-5$ are also proved in section 7 .

\section{Graphs with large number of edges}

Let $k \geqq 2$ and $\alpha \geqq 0$ be real numbers and let

$$
\mathscr{D}(k, \alpha) \doteq\left\{G=(V, E):|V| \geqq 3,|E|>k \cdot|V|+\alpha \cdot|V|^{2}-1\right\} .
$$

Lemma 1. Suppose that $k \geqq 2$ and $\alpha \geqq 0$ are fixed real numbers and the graph $G=$ $=(V, E) \in \mathscr{D}(k, \alpha)$ cannot be contracted to any graph in $\mathscr{D}(k, \alpha)$. Then every edge of $G$ is in more than $k+2 \alpha \cdot|V|-\alpha-1$ triangles.

Proof. Let $k \geqq 2$. If $n=3$ then $|E| \geqq 2 \cdot 3-1=5$ and this is impossible. Hence $n>3$. Suppose that an edge $(u, v)$ of $G$ is contained by at most $k+2 \alpha n-\alpha-1$ triangles. Let $G^{\prime}=\left(V^{\prime}, E^{\prime}\right)$ denote the graph obtained from $G$ by the contraction of the edge $(u, v)$. Then $\left|V^{\prime}\right|=n-1 \geqq 3$ and $\left|E^{\prime}\right| \geqq|E|-(k+2 \alpha n-\alpha-1)-1 \geqq k n+\alpha n^{2}-1-k-$ $-2 \alpha n+\alpha=k(n-1)+\alpha(n-1)^{2}-1$. Thus $G^{\prime} \in \mathscr{D}(k, \alpha)$, a contradiction.

Lemma 2. If $k \geqq 39$ then every graph $G \in \mathscr{D}(k, 1 / 3)$ can be contracted to a graph $G_{0}=\left(V_{0}, E_{0}\right)$ such that $\left|V_{v}\right| \geqq 5 k+1$ and $\delta\left(G_{0}\right) \geqq 7 / 8 \cdot\left|V_{0}\right|$.

Proof. Suppose that the lemma is not true. Let $G=(V, E)$ be a counterexample with minimum number of edges and let $|V|=n$. Then $|E| \leqq k n+n^{2} / 3$. Since $n k+n^{2} / 3-$ $-1<\left(n^{2}-n\right) / 2$ so

$$
n \geqq 6 k+1
$$

Specially, since $k \geqq 39$ so

$$
n \geqq 235
$$


If $\delta(G) \geqq 7 n / 8$ then $G_{0}=G$ provides the statement of the lemma, a contradiction to the choice of $\mathrm{G}$. Therefore

$$
\delta(G)<7 n / 8
$$

Let $v_{0} \in V, d_{G}\left(v_{0}\right)=\delta(G)$. Consider the graph $G^{\prime} \doteq G\left(N_{G}\left(v_{0}\right) \cup\left\{v_{0}\right\}\right)$. For every vertex $v \in N_{G}\left(v_{0}\right)$, the edge $\left(v_{0}, v\right)$ is in more than $k+2 n / 3-1 / 3-1$ triangles by Lemma 1 , i.e. $\delta\left(G^{\prime}\right)>k+2 / 3-1 / 3$. Thus if $8 / 7(k+2 / 3-1 / 3) \geqq 1+\delta(G)$ then for $G_{0}=G^{\prime}=\left(V^{\prime}, E^{\prime}\right)$, we have $\left(V^{\prime} \mid \geqq k+2 / 3 n-1 / 3+1 \geqq k+4 k+2 / 3+2 / 3>5 k+1\right.$, a contradiction to the choice of $G$. Thus

$$
\frac{8}{7}\left(k+\frac{2}{3} n-\frac{1}{3}\right)<1+\delta(G) \text {. }
$$

Then

and hence

$$
\frac{7}{8}\left(k+\frac{2}{3} n-\frac{1}{3}\right)<1+\frac{2|E|}{n} \leqq 1+2+\frac{2}{3} n \text {. }
$$

$$
k>\frac{n}{9}-\frac{29}{18} .
$$

Combining the inequalities (3), (4) and (5), we get $n<232$, a contradiction to (2).

Lemma 3. If $k \geqq 8$ then every graph $G \in \mathscr{D}(k, 2 / 9)$ can be contracted to a graph $G_{0}=$ $=\left(V_{0}, E_{0}\right)$ such that $\left|V_{0}\right| \geqq 13 k / 5$ and $\delta\left(G_{0}\right) \geqq 3\left|V_{0}\right| / 4$.

Proof. Suppose that the lemma is not true. Let $G=(V, E)$ be a counterexample with minimum number of edges and let $|V|=n$. As in the proof of Lemma 2, we obtain the following inequalities:

$$
\begin{gathered}
n \geqq \frac{18}{5} k \\
n \geqq 29 \\
\delta(G)<\frac{3}{4} n \\
\frac{4}{3}\left(k+\frac{4}{9} n-\frac{2}{9}\right)<1+\delta(G) \\
k>\frac{2}{9} n-\frac{35}{18} .
\end{gathered}
$$

Combining the inequalities $\left(3^{\prime}\right),\left(4^{\prime}\right)$ and $\left(5^{\prime}\right)$, we get $n<14$, a contradiction to $\left(2^{\prime}\right)$.

Lemma 4. Let $k \geqq 6480$ be a natural number and let $G \in \mathscr{E}_{k}$ be a graph where $\mathscr{E}_{k}=$ $=\left\{G=(V, E):|V| \geqq k,|E|>k \cdot|V|-\left(\begin{array}{c}k+1 \\ 2\end{array}\right)\right\}$. Then $G$ can be contracted to a graph $H=\left(V_{0}, E_{0}\right)$ such that $\left|V_{0}\right| \geqq 13 k / 432+1$ and $\delta(H) \geqq 7\left|V_{0}\right| / 8$. 
Proof. Let $G_{1}=\left(V_{1}, E_{1}\right)$ be a graph in $\mathscr{E}_{k}$ with minimum number of edges that $G$ can be contracted onto. Then $\left|E_{1}\right|<k \cdot\left|V_{1}\right|$ and $\delta\left(G_{1}\right) \leqq 2 k-1$. Let $v_{0} \in V_{1}$ be a vertex such that $\delta_{G_{1}}\left(v_{0}\right)=\delta\left(G_{1}\right)$ and $G_{2}\left(V_{2}, E_{2}\right) \doteq G_{1}\left(N_{G_{1}}\left(v_{0}\right) \cup\{v\}\right)$. As in the proof of Lemma 1 , it is easy to see that every edge in $E_{1}$ is contained by more than $k-1$ triangles. Thus $\delta\left(G_{2}\right)>k$. Notice that $\left|V_{2}\right|=\delta\left(G_{1}\right)+1 \leqq 2 k$ and $\left|E_{2}\right| \geqq 1 / 2 \delta\left(G_{2}\right)\left|V_{2}\right| \geqq$ $\geqq k\left|V_{2}\right| / 2$.

Let $k_{1} \doteq k / 18$. Then $G_{2} \in \mathscr{D}\left(k_{1}, 2 / 9\right)$. According to Lemma $3, G_{2}$ can be contracted to a graph $G_{3}=\left(V_{3}, E_{3}\right)$ such that $\left|V_{3}\right| \geqq 13 k_{1} / 5=13 k / 90$ and $\delta\left(G_{3}\right) \geqq 3\left|V_{3}\right| / 4$ Let $k_{2} \doteq\left|V_{3}\right| / 24$. Then $G_{3} \in \mathscr{D}\left(k_{2}, 1 / 3\right)$ and according to Lemma $2, G_{3}$ can be contracted to a graph $H=\left(V_{0}, E_{0}\right)$ such that $\left|V_{0}\right| \geqq 5 k_{2}+1 \geqq 5 / 24\left|V_{3}\right|+1 \geqq 13 k / 24 \cdot 18 k+1$ and $\delta(H) \geqq 7\left|V_{0}\right| / 8$.

So Lemma 4 implies that to prove the inequality $\min _{G \in \mathcal{E}_{k}} \eta(G) \geqq c_{1} k / \sqrt{\log k}$ it is sufficient to show that $\eta(G) \geqq c_{2} n / \sqrt{\log n}$ holds for any graph $G=(V, E)$ with $|V|=4$ and $\delta(G) \geqq 7 n / 8$.

\section{Contraction of graphs with large number of edges}

Lemma 5. $\eta(\bar{G}) \geqq 3|V| / 4-1 / 4$ holds for any graph $G=(V, E)$ with $|E|<|V| / 2$.

Proof. Let $G=(V, E)$ be a counterexample with the minimum number of vertices and lat $|V|=n$. It is obvious that $n>4$. We distinguish three cases.

Case 1. $\sigma(G) \geqq 2$.

Let $d_{G}\left(v_{1}\right)=\delta(G)$ and $G_{1}=\left(V_{1}, E_{1}\right) \doteq G-\left\{v_{1}\right\}$. Then $2\left|E_{1}\right|<n-4 \mid=$ $=\left|V_{1}\right|-3$ and there exist isolated vertices $v_{2}, v_{3}, v_{4}$ in $G_{1}$. Let $G_{2} \doteq G_{1}-\left\{v_{1}, v_{3}, v_{4}\right\}$. Then $\eta\left(\bar{G}_{2}\right) \geqq(3 / 4)(n-4)-1 / 4=3 n / 4-13 / 4$ by the minimality of $G$. Since the vertices $v_{2}, v_{3}, v_{4}$ are pairwise adjacent in $\bar{G}_{1}$ so $\eta(\bar{G}) \geqq \eta\left(\bar{G}_{1}\right) \geqq 3+\eta\left(\bar{G}_{2}\right) \geqq 3 n / 4-1 / 4$, a contradiction to the choice of $G$.

Case 2. $|E| \leqq 1$.

Then $\eta(\bar{G}) \geqq n-1 \geqq 3 n / 4-1 / 4$.

Case 3. $\sigma(G)=1,|E| \geqq 2$.

Let $e_{1}=\left(v_{1}, v_{2}\right) \in E$ and $e_{2}=\left(v_{3}, v_{4}\right) \in E$. Contracting the edge $\left(v_{1}, v_{3}\right)$ in the graph $\bar{G}$, we obtain a graph $\bar{G}_{1}=\left(V_{1}, \bar{E}_{1}\right)$ with $\left|V_{1}\right|=n-1$ in which the vertices $v_{2}, v_{4}$ and the vertex $\tilde{v}$ obtained from $v_{1}$ and $v_{3}$ are adjacent to each other. Then $\eta\left(\bar{G}-\left\{v_{1}, v_{2}, v_{3}, v_{4}\right\}\right) \geqq(3 / 4)(n-4)-1 / 4=3 n / 4-13 / 4$ by the minimality of $G$. Hence $\eta(\bar{G}) \geqq 3+(3 n / 4-13 / 4)=3 n / 4-1 / 4$.

Lemma 6. (crucial one) Let $r \geqq G$ and $G=(V, E)$ be a graph of nertices such that $\sigma(G) \leqq n / r$. Then $\bar{G}$ can be contracted to a graph $\bar{H}=\left(V_{0}, \bar{E}_{0}\right)$ of $|n / 2|$ vertices such that $2\left|E_{0}\right| \leqq\left(27 r /(r-2)^{3}(r-1)^{2}\right) \cdot\left|V_{0}\right|^{2}$.

Proof. Connect every vertex $v \in V$ by a quasi-edge to the vertices which are not adjacent to $v$ and from which more than $3 n /(r-1)(r-2)$ paths of length two lead to $v$. We show that every vertex is incident to at most $(n(r-2) / 3 r)-1$ quasi-edges. If it is not the case then the number of paths of length two from $v$ is greater than $(3 n /(r-1)(r-2))(n(r-2)-3 r) / 3 r$. At the same time, the total number of these paths 
is at most $\sigma(G)(\sigma(G)-1) \leqq n(n-r) / r^{2}$ and the inequality

$$
\frac{3 n}{(r-1)(r-2)} \cdot \frac{n(r-2)-3 r}{3 r}>\frac{n(n-r)}{r^{2}}
$$

holds for any natural number $n \geqq r \geqq G$.

Let $G^{\prime}=\left(V^{\prime}, E^{\prime}\right)$ denote the graph obtained from $G$ by adding the quasi-edges. Then $\sigma\left(G^{\prime}\right) \leqq n / r+(n(r-2) / 3 r)-1=\left(n(r+1 / 3 r)-1<(n-1) / 2\right.$, i.e. $\bar{G}^{\prime}$ has a Hamiltonian cycle and a matching of $[n / 2]$ edges.

The contraction of an edge $\left(v_{1}, v_{2}\right)$ in $\bar{G}$ corresponds to a sticking in $G$ : we replace the non-adjacent vertices $v_{1}$ and $v_{2}$ with a new vertex $v_{3}$ adjacent to $w \in V-$ $-\left\{v_{1}, v_{2}\right\}$ iff $\left(v_{1}, w\right) \in E$ and $\left(v_{2}, w\right) \in E$.

Let $\pi$ be an arbitrary matching of $\lfloor n / 2\rfloor$ edges in $\overline{G^{\prime}}$. Sticking every pair of vertices in $G$ corresponding to the edges of $\pi$ and deleting the vertex not covered by $\pi$ if $n$ is odd, we obtain a graph $H_{\pi}$ of $[n / 2]$ vertices. From among the graphs $H_{\pi}$, we choose a graph $H \doteq H_{\pi_{0}}=\left(V_{0}, E_{0}\right)$ with minimum number of edges. Let $V_{0}=$ $=\left\{v_{1}: i=1, \ldots, n / 2\right\}$ and suppose that $v_{i}$ is obtained by sticking $v_{1 i}$ and $v_{2 i}$. Notice that $\left(v_{i}, v_{j}\right) \in E_{0}$ iff $\left\{\left(v_{1 i}, v_{1 j}\right),\left(v_{1 i}, v_{2 j}\right),\left(v_{2 i}, v_{1 j}\right),\left(v_{2 i}, v_{2 j}\right)\right\} \subset E$.

A path $\left(x_{1}, x_{3}, x_{2}\right)$ of length 2 will be called a fork on the vertex pair $\left\{x_{1}, x_{2}\right\}$. We will say that the pair $\left\{x_{1}, x_{2}\right\}$ is the support of this fork.

If $\left(v_{1 i}, v_{1 j}\right) \notin E^{\prime}$ and $\left(v_{2 i}, v_{2 j}\right) \notin E^{\prime}\left(\left(v_{1 i}, v_{2 j}\right) \notin E^{\prime}\right.$ and $\left(v_{2 i}, v_{1 j}\right) \notin E^{\prime}$, resp.) (for some $i, j \in\{1, \ldots, 1 / 2\}, i \neq j$ then the graph $H_{i j}=\left(V_{i j}^{1}, E_{i j}^{1}\right)\left(H_{i j}^{2}=\left(V_{i j}^{2}, E_{i j}^{2}\right)\right.$, resp. $)$ is called the $(i, j, 1)$-transformation $((i, j, 2)$-transformation, resp.) of the graph $H_{0}=\left(E_{0}, V_{0}\right)$. The graph $H_{i j}^{1}\left(H_{i j}^{2}\right.$, resp. $)$ is obtained by stickings along the matching $\pi_{l j}^{1}\left(\pi_{i j}^{2}\right.$, resp.) in $\overline{G^{\prime}}$, where $\pi_{i j}^{1}\left(\pi_{i j}^{2}\right.$, resp. $)$ is obtained from $\pi_{0}$ replacing the edges $\left(v_{1 l}, v_{2 i}\right)$ and $\left(v_{1 j}, v_{2 j}\right)$ with the edges $\left(v_{1 i}, v_{1 j}\right)$ and $\left(v_{2 i}, v_{2 j}\right)\left(\left(v_{1 i}, v_{2 j}\right)\right.$ and $\left(v_{2 i}, v_{1 j}\right)$, resp.). These transformations will be called $(i, j)$-transformations.

Let $d_{H}\left(v_{i}\right)=t_{i}(1 \leqq i \leqq[n / 2])$. During an $(i, j, l)$-transformation, $t_{i}+t_{j}$ edges of $E_{0}-E_{i j}^{l}$ are destroyed but it may occur that new edges arise. The edges of $G^{\prime}$ hinder the existence of some $(i, j)$-transformations. Since every edge of $G^{\prime}$ may cross at most one transformation so every vertex $v_{i} \in V$ can take part in at least $n-3-2\left(\frac{n(r+1)}{3 r}-1\right)$ transformations. Then $\left|E_{i j}^{l}-E_{0}\right| \geqq t_{1}+t_{0}$ holds for $1 \leqq i, j \leqq$ $\leqq[n / 2], 1 \leqq l \leqq 2$ by the choice of $H$. Every edge of $E_{i j}^{l}-E_{0}$ has a pair of forks in $G$ with common supports in $\pi_{0} \cap \pi_{i j}^{l}$. Every pair of forks may take part in at most one transformation producing "new" edge. Then every $(i, j, l)$-transformation corresponds to $x_{i j}^{l} \geqq t_{i}+t_{j}$ pairs of forks and the members of every such pair have a common support belonging to $\pi_{0}$. Associate $t_{i}$ pairs from among these $x_{i j}^{l}$ pairs of forks with $v_{i}$ and $t_{j}$ pairs from among the remaining $x_{i j}^{l}-t_{i}$ pairs with $v_{j}$. Then at least $t_{i}(n-(2 n(r+1) / 3 r)-1)$ pairs of forks will belong to $v_{i}(1 \leqq i \leqq[n / 2])$. Furthermore, every edge $\left(v_{i}, v_{j}\right) \in E_{0}$ corresponds to the pairs $\left\{\left(v_{1 i}, v_{1 j}, v_{2 j}\right)\left(v_{1 i}, v_{2 j}, v_{2 i}\right)\right\}$ and $\left\{\left(v_{1 j}, v_{1 l}, v_{2 j}\right),\left(v_{1 j}, v_{21}, v_{2 j}\right)\right\}$ of forks not belonging to any vertex. I.e., the total number of pairs of forks in $G$ with common supports in $\pi_{0}$ is at least

$$
\sum_{i=1}^{\left\lfloor\frac{n}{2}\right\rfloor} t_{i}\left(\frac{n(r-2)}{3 r}-1\right)+2\left|E_{0}\right|=\left(\frac{n(r-2)}{3 r}-1\right) \cdot 2\left|E_{0}\right|+2\left|E_{0}\right|=2\left|E_{0}\right| \frac{n(r-2)}{3 r} \text {. }
$$


On the other hand, $\left(v_{1 i}, v_{2 i}\right) \in E^{\prime}$ for $1 \leqq i \leqq\left[n / 2 \mid\right.$ thus $\left\{v_{1 i}, v_{2 i}\right\}$ is the support of at most $(3 n /(r-1)(r-2))$ forks and so it is the common support of at most $\left(\frac{3 n}{(r-1)(r-2)}\right)$ pairs of forks. Hence

$$
\begin{gathered}
\frac{2\left|E_{0}\right| n(r-2)}{3 r} \leqq\left\lfloor\frac{n}{2}\right\rfloor \frac{9 n}{2(r-1)^{2}(r-2)^{2}} \cdot\left(n-\frac{(r-1)(r-2)}{3}\right), \text { i.e. } \\
2\left|E_{0}\right| \leqq\left\lfloor\frac{n}{2}\right\rfloor \cdot \frac{n-1}{2} \cdot \frac{27 r}{(r-1)^{2}(r-2)^{3}} .
\end{gathered}
$$

\section{Lower estimation of $\eta(\bar{G})$}

Let $f(n, m) \doteq n / 2 \sqrt{\log n}\left(\sqrt{\log n^{2} / 2 m}-14\right) \quad$ and $f(G) \doteq f(|V|,|E|)$ for any graph $G=(V, E)$. The aim of this section is to give a lower bound of $\eta(\bar{G})$ by $f(G)$ for graphs with small ratio $|E| /|V|^{2}$.

Lemma 7. Let $n \geqq 3, \quad 2 m \geqq n, \quad 1.4<\sqrt{\log n^{2} / 2 m}$ and $n / 2>\alpha \geqq 1+\log n^{2} / 2 m-$ $-1.4 \sqrt{\log n^{2} / 2 m}$. Then $f(n, m) \leqq f(n-1, m-2 m \alpha / n)$.

Proof. Let $c \doteq n^{2} / 2 m, c_{1} \doteq(n-1)^{2} / 2(m-(2 m \alpha / n))$. Then

$$
f\left(n-1, m-\frac{2 m \alpha}{n}\right)-f(n, m) \geqq \frac{1}{2 \sqrt{\log n}}\left((n-1)\left(\sqrt{\log c_{1}}-1.4\right)-n(\sqrt{\log c}-1.4)\right) .
$$

I.e., it is sufficient to prove the inequality

$$
(n-1) \sqrt{\log c_{1}} \geqq n y^{\prime} \overline{\log c}-1.4 \text {. }
$$

The inequality for $\alpha$ in the conditions of the lemma implies that

Since $x<\log (1-x)^{-1}$ for $x \in(0,1)$ so

$$
2 \log c-2.8 \sqrt{\log c} \equiv 2(\alpha-1) \cdot \frac{(n-1)^{2}}{n(n-2)} .
$$

$$
\begin{aligned}
2(\alpha-1) \frac{(n-1)^{2}}{n(n+2)} & \leqq \frac{(n-1)^{2}}{n} \log \left(1-\frac{2(\alpha-1)}{n-2}\right)^{-1}=\frac{(n-1)^{2}}{n} \cdot \log \frac{n-2}{n-2 \alpha} \leqq \\
& \leqq \frac{(n-1)^{2}}{n} \cdot \log \frac{\left(1-\frac{1}{n}\right)^{2}}{1-\frac{2 \alpha}{n}}=\frac{(n-1)^{2}}{n} \cdot\left(\log \frac{n^{2}\left(1-\frac{1}{n}\right)^{2}}{2 m\left(1-\frac{2 \alpha}{n}\right)}-\log \frac{n^{2}}{2 m}\right)= \\
& =\frac{(n-1)^{2}}{n}\left(\log c_{1}-\log c\right) .
\end{aligned}
$$

Thus

$$
(n-1)^{2}\left(\log c_{1}-\log c\right) \geqq 2 n \log c-2.8 n \sqrt{\log c} .
$$


Then

$$
\begin{gathered}
(n-1)^{2} \log c_{1} \geqq \\
\geqq(n-1)^{2} \log c+2 n \log c-2.8 n \sqrt{\log c}=n^{2} \log c+\log c-2.8 n \sqrt{\log c}= \\
=(n \sqrt{\log c}-1.4)^{2}+(\log c-1.96)>(n \sqrt{\log c}-1.4)^{2},
\end{gathered}
$$

i.e. (6) holds.

With the help of derivates, it is easy to prove

Lemma 8. If $x \geqq \sqrt{\log 49}$ then $e^{1.4 x} / 5 \geqq 1+x^{2}-1.4 x$.

Lemma 9. Let $G=(V, E)$ be a graph with $|V|=n, E \neq \emptyset$ and suppose that $c \doteq n^{2} / 2|E| \geqq$ $\geqq 49, \sigma(G) \leqq n / c \cdot(1 / 5) e^{1.4 \sqrt{\log c}}$. Then $\bar{G}$ can be contracted to a graph $\bar{H}=\left(v_{0}, \bar{E}_{0}\right)$ of $[n / 2\rfloor$ vertices such that either $E_{0}=\emptyset$ or $f(H) \geqq f(G)$.

Proof. Let $z \doteq \sigma(G) c / n$. According to Lemma $6, \bar{G}$ can be contracted to a graph $\bar{H}=\left(V_{0}, \bar{G}_{0}\right)$ such that $\left|V_{0}\right|=[n / 2]$ and either $E_{0}=\emptyset$ or $c_{1} \doteq\left|V_{0}\right|^{2} / 2\left|E_{0}\right| \geqq(c / z)^{4}$. $\cdot 1 / 27 \cdot(1-3 / c)^{2}(1-23 / c)^{3}$. Suppose that $E_{0} \neq \emptyset$. If $c \geqq 49$ then $(1 / 5 c) e^{1.4 \gamma \overline{\log c}}<1 / 15$ and if $z / c<1 / 15$ holds then $(1-z / c)^{2}(1-2 z / c)^{3}>43 / 76$. Thus

$$
c_{1} \geqq \frac{43}{76 \cdot 27} \cdot\left(\frac{c}{z}\right)^{4}>0.02\left(\frac{c}{z}\right)^{4} .
$$

Estimate $f(H)-f(G)$.

$$
\begin{gathered}
f(H)-f(G)=\frac{\left\lfloor\frac{n}{2}\right\rfloor\left(\sqrt{\log c_{1}}-1.4\right)}{2 \sqrt{\log }\left\lfloor\frac{n}{2}\right\rfloor}-\frac{n(\sqrt{\log c}-1.4)}{2 \sqrt{\log c}} \geqq \\
\geqq \frac{n}{2 \sqrt{\log n}}\left(\frac{1}{2} \sqrt{\log c_{1}}-\sqrt{\log c}+0.7\right)=\frac{n}{2 \sqrt{\log n}} \frac{0.25 \log c_{1}-(\sqrt{\log c}-0,7)^{2}}{0.5 \sqrt{\log c_{1}}+\sqrt{\log c}-0.7} .
\end{gathered}
$$

I.e., it is sufficient to prove the inequality

$$
\frac{1}{4} \log c_{1}-\log c+1.4 \sqrt{\log c}-.49 \geqq 0 .
$$

Applying (7), the left side of (8) is at least

$$
\log c-\log z+\frac{1}{4} \log .02-\log c+1.4 \sqrt{\log c}-0.49 \geqq 1.4 \sqrt{\log c}-\log \left(z \cdot 50^{1 / 4} e^{1 / 2}\right) .
$$

We have $z \leqq e^{1.4 \sqrt{\log c}} / 5<e^{1.4 \sqrt{\log c}} / \sqrt[4]{50 e^{2}}$ by the conditions of the lemma and so (8) holds.

Lemma 10. The inequality $\eta(\bar{G}) \geqq f(G)$ holds for any graph with $E \neq \emptyset$ and $|V|^{2} / 2|E| \geqq$ $\geqq 49$. 
Proof. Let $|V|=n, c \doteq|V|^{2} / 2|E|, z=\sigma(G) \cdot c / n$. If $n \leqq 8$ then the statement of the lemma is obvious. Suppose that the lemma is true for $n^{\prime}<n$. We distinguish four cases.

Case $1.0<2|E|<n$.

If $|E|>0$ then $f(G) \leqq n \sqrt{\log n^{2}} / 2 \sqrt{\log n}=(\sqrt{2} / 2) n$ and $\eta(\bar{G}) \geqq 3 n / 4-1 / 4 \geqq$ $\geqq(\sqrt{2} / 2) n$ by Lemma 5 .

Case 2. $2|E| \geqq n, z \leqq e^{1.4 \sqrt{\log c} / 5}$.

Since $c \leqq n$ so $f(G) \leqq[n / 2]$. Let $H=\left(V_{0}, E_{0}\right)$ be a graph described in Lemma 9. If $E_{0}=\emptyset$ then $\eta(\bar{G}) \geqq[n / 2] \geqq f(G)$. If $E_{0} \neq \emptyset$ then $f(H) \geqq f(G)$ and the characteristics of the function $f$ imply that $\left(\left|V_{0}\right|^{2} / 2\left|E_{0}\right|\right)>c \geqq 49$. Then $\eta(\bar{H}) \geqq f(H) \geqq f(G)$ by the induction hypothesis. But $\eta(\bar{G}) \geqq \eta(\bar{H})$.

Case 3. $2|E| \geqq n, z \geqq 1+\log c-1.4 \sqrt{\log c}$.

Deleting a vertex $v_{0}$ with $d_{G}\left(v_{0}\right)=\sigma(G)=z n / c$ from $G$, we obtain a graph $G^{\prime}=\left(V^{\prime}, E^{\prime}\right)$ with $\left|V^{\prime}\right|=n-1$ and $\left|E^{\prime}\right| \leqq|E|-2|E| / n(1+\log c-1.4 \sqrt{\log c})$. Now $f\left(G^{\prime}\right) \geqq f(G)$ by Lemma 7 . It is clear that $\left|V^{\prime}\right|^{2} / 2\left|E^{\prime}\right| \geqq c \geqq 49$. And $\eta\left(\overline{G^{\prime}}\right) \geqq f\left(G^{\prime}\right) \geqq$ $\geqq f(G)$ by the induction hypothesis.

Thus, we have to settle only

Case 4. $2|E| \geqq n, 1+\log c-1.4 \sqrt{\log c}>z>e^{1.4 \sqrt{\log c}} / 5$.

If $c \geqq 49$ then $e^{1.4 \sqrt{\log c} / 5} \geqq 1+\log c-1.4 \sqrt{\log c}$ holds by Lemma 8 .

\section{Proof of Theorem 1' and its corollaries}

\section{Proof of Theorem 1'.}

Case 1. $k \leqq 6480$.

Mader [5] proved that $\eta_{1}(k) \geqq 1 / 8\left(k / \log _{2} k\right)$ and if $k \leqq 6480$ then $(1 / 8)(k / \log k) \geqq(1 / 270)(k / \sqrt{\log k})$ holds.

Case 2. $k>6480$.

Let $G \in \mathscr{E}_{k}$. Then $G$ can be contracted to a graph $H=\left(V_{0}, E_{0}\right)$ such that $\left|V_{0}\right| \geqq 13 k / 432+1$ and $\sigma(\bar{H}) \leqq\left|V_{0}\right| / 8$ by Lemma 4 . Now $H$ can be contracted to a graph $H_{1}=\left(V_{1}, E_{1}\right)$ such that $\left|V_{1}\right| \geqq 13 k / 864$ and $2\left|E_{1}\right| \leqq\left|V_{1}\right|^{2} / 49$ by Lemma 6 . Let $c \doteq\left(\left|V_{1}\right|^{2} / 2\left|E_{1}\right|\right)$ if $\bar{E}_{1} \neq \emptyset$. Then

$$
\eta\left(H_{1}\right) \geqq f\left(\bar{H}_{1}\right)=\frac{(\sqrt{\log c}-1.4)|| v_{1} \mid}{2 \sqrt{\log \left|v_{2}\right|}} \geqq \frac{.5\left|v_{1}\right|}{2 \sqrt{\log \left|v_{1}\right|}} \geqq \frac{1}{4} \cdot \frac{13 k}{864} \cdot \frac{1}{\sqrt{\log k}} \geqq \frac{k}{270 \sqrt{\log k}}
$$

by Lemma 10 . Finally if $\bar{E}_{1}=\emptyset$ then $\eta(G) \geqq\left|V_{1}\right| \geqq 13 / 864 k$.

Proof of Corollary 2. If $k \leqq 1000$ then the statement holds obviously. Let $k>1000$. Every $k$-chromatic graph $G$ contains a critical $k$-chromatic subgraph $G^{\prime}$ such that the 
degree of every vertex of $G^{\prime}$ is at least $k-1$. It is clear that $G^{\prime} \in \mathscr{D}_{\left[\frac{k-1}{2}\right]}$. Then $\eta(G) \geqq$ $\geqq \eta\left(G^{\prime}\right) \geqq 1 / 270 \cdot \frac{\left[\frac{k-1}{2}\right\rfloor}{\sqrt{\log k / 2}} \geqq k / 540 \sqrt{\log k}$ by Theorem 1 .

Proof of Corollary 3. It is known that $\chi(G) \leqq n / \log n$ holds for almost all graphs $G$ with $n$ vertices (see [3]). Furthermore, it it easy to see that $|E| \geqq n^{2} / \delta$ for almost all graphs $G=(V, E)$ with $n$ vertices. Then $\eta(G) \geqq 1 / 270 \cdot(n / 6) / v \sqrt{\log n})$ by Theorem 1 . If, in addition $\sqrt{\log n}>6 \cdot 270$ then $\chi(G) \leqq \eta(G)$.

Proof of Corollary 4. It is known (see [1], p. 96) that if $k$ is large enough then $\chi(G) \leqq 3 k / \log k$ holds for almost all graphs $G$ of $n$ vertices and $k n$ edges. Now $\eta(G) \geqq(1 / 270)(k / \sqrt{\log k})$ for any graph $G$ of this class by Theorem 1 . Therefore if $k$ is large enough then Corollary 4 is true.

Proof of Corollary 5. Let $G=(V, E)$ be an arbitrary graph with $|V|=n$. Then either $|E| \geqq(n-1) n / 4$ or $|\bar{E}| \geqq(n-1) n / 4$. Now $\max \{\eta(G), \eta(\bar{G})\} \geqq$ $\geqq(1 / 270)(n / 4 \sqrt{\log n})$ by Theorem 1 .

On the other hand, as we said it in the introduction, the Hadwiger number of almost all graphs of $n$ vertices is at most $n / \sqrt{\log n}$. Thus if $n$ is large then there exists a graph $G_{0}=\left(V_{0}, E_{0}\right)$ with $\left|V_{0}\right|=n$ such that $\eta\left(G_{0}\right) \leqq n / \sqrt{\log n}$ and $\eta\left(\bar{G}_{0}\right) \leqq$ $=n / \sqrt{\log n}$. Then $\eta\left(G_{0}\right)+\eta\left(\bar{G}_{0}\right) \leqq 2 n / \sqrt{\log n}$.

\section{References}

[1] P. ERdös, J. Spencer, Probabilistic methods in combinatorics, Academic Press, New YorkLondon, 1974.

[2] H. HADWIGER, Über eine Klassifikation der Streckenkomplexe, Viert. Naturforsch. Ges. Zürich, 88 (1943), 133-142.

[3] A. D. Korshunov, On the chromatic number of graphs on $n$ vertices (in Russian), Metody diskretnogo analiza $v$ teorii bulevyh finksij i skhem, 35 (1980), 15-45.

[4] A. V. KostochKa, On the minimum Hadwiger number of graphs with given average degree (in Russian), submitted to Diskretnij Analiz.

[5] W. MADER, Homomorphiesätze für Graphen, Math. Annalen, 178 (1968), 154-168.

[6] Z. Miller, Contractions of graphs: A theorem of Ore and an extremal problem, Discrete Math., 21 (1978), 261-273.

[7] K. WAGNER, Beweis einer Abschwächung der Hadwiger-Vermutung, Math. Annalen, 153 (1964), $139-141$.

[8] B. ZeLINKA, On some graph-theoretical problems of V. G. Vizing, Cas. Pestov. Math., 98 (1973), $56-66$.

[9] A. A. ZYKov, On the edge number of graphs with no greater Hadwiger number than 4, Prikladnaja matematika i programmirovanije, 7 (1972), 52-55.

\section{A. V. Kostochka}

630090 Novosibirsk-90

Mathematical Institute of the

Siberian Branch of the

Soviet Academy of Sciences

U.S.S.R. 